\title{
SONAR: Communication System for Supporting Information Gathering and Social Interaction in a Niche Market
}

\author{
Junichi Hoshino, Takeru Umemura, Sachi Urano, and Daiki Satoi \\ University of Tsukuba, 1-1-1-\#3M309 Tennodai Tsukuba, Ibaraki 305-8573, Japan \\ jhoshino@esys.tsukuba.ac.jp \\ http://www.entcomp.esys.tsukuba.ac.jp/
}

\begin{abstract}
We propose a new communication system by which niche people can obtain cross-cutting information and communicate with other people based on each personality. The system graphically displays the degree and direction of other people's hobbies who are interested in the keyword niche people input, and relation between the knowledge e.g. movies, music, animation, history, geography using nodes. So, we can search friends who have similar interest and direction in hobbies. From the demonstration experiments, we obtained good results that the system could help niche people to gain and exchange useful information.
\end{abstract}

Keywords: Niche, Communication System, Cross-Cutting Information.

\section{Introduction}

Hobbies, while they enrich our daily lives, also play a large role in the economy. Having a hobby is also an important activity from the health perspective, as having a hobby is one criterion used to measure health[1][2], and people with a hobby are found to be three times less likely than people without a hobby to contract liver-related diseases[3]. The recent trend - brought to us by technological advancements that allow us to spend our spare time in more ways than ever - is an increase in people with "niche" interests, especially among the younger population, that immerse in entertainment fields that resonate with their values[4]. Therefore, there is an increasing demand for a new system that allows for active information gathering by the users themselves; the uniformity of information provided by mass media has increasingly limited value.

Existing online services that offer information related to hobbies are not useless, with their efforts to make information in demand more accessible through implementation of search categories (movie, music, anime, etc.) as well as selective suggestions based on specific content accessed by the user. However, the users' needs for niche information, as well as communication and exchange of information with other users, has been given very little consideration.

On the other hand, there are services focused on supporting inter-user communication for acquisition of information. Social networking services, such as mixi and Quora, allow users to join communities based on interests and comment on topics created by other users. However, even within these communities, the direction and depth of the 
users' interests vary greatly, hindering dialogue between commenting users, and making it difficult to gain information that matches the particular user's interests.

Online forums such as 2 Channel and 4chan do a better job of inducing communication with their interface, making it easy to access cross-boundary information based on the user's interests. Yet, the linear format of these threads makes it difficult to discern one conversation from another, obscures the topic itself, and deteriorates the utility and relativity of the information posted. As a result, niche users are forced to gather information through repeatedly searching with clever keywords or comparing multiple websites. Having both users superficially interested in trendy topics and users with indepth knowledge on specific topics also causes miscommunication, misunderstandings, and sometimes arguments, wasting precious time that could be spent on the hobby itself to fruitless conversation.

As a means to visually support acquisition of information, visualization experts are researching query development using Boolean algebra and Venn diagrams[5], "VRVIBE," a system that displays clusters of documents related to the user's interests[6], "Vizster," a service that graphically illustrates the connections between SNS users[7], "VISTORY," which balances public and private events on the same timeline [8], among others. There are also services that facilitate acquisition of information by clever display of information, such as Document Lens[9], Perspective Wall[10], Graphical Fisheye View[11], and TreeMap[12][13]. However, these research projects do not pay enough consideration to visualizing users' unique interests or supporting exchange of information based on user interests.

In this article, I will make a proposal of a communication system that allows users with niche interests to share information with each other, while also employing visualization to facilitate acquisition of knowledge across boundaries. This system will graphically display specific information about other users' interests, including direction and depth, and show how this information relates to the user's knowledge on the topic through nodes and timelines. By displaying the current interests of people with similar direction and depth of interest in a topic, as well as acquiring and sharing detailed information on that topic, this system aims to facilitate social interaction.

\section{Discussion of System Design}

Consideration to the requirements of a communication system that facilitates hobbyrelated activity brings us to two conclusions: the necessity of a means to help users acquire detailed information in multiple fields, as well as easily understandable representation of the relation of this information to the users themselves.

In this system, visual representation of individual users' specific interests will be achieved by colored beams radiating from circular user nodes (representing individual users), whose color and length will change according to niche, as well as visualizing time spent on the interest by variation in the size of the circle. A list of the top 10 interests of the user will also be displayed. By referencing this information during conversations, users will be able to better understand whether their counterpart is widely interested in trendy topics or is deeply interested in a niche, as well as know how much knowledge they have on the topic. Visualizing interests in this manner will reduce 
awkward conversation and facilitate meaningful exchange by allowing users to select to engage other users with interest characteristics especially meaningful to them.

The user nodes will display the ratio of interest within a topic by the length of up to 12 colored beams (representing subgenres) and the amount of knowledge of the topic by the size of the gray circle (Fig. 1). The colors of the beams are selected based on the PCCS (Practical Color Co-ordinate System), and the beams are placed clockwise around the node starting with red at 12 o'clock, with intervals between beams at $360 /[$ no. subgenres] degrees. In the case of music, the ratio will be [no. plays within genre]/[total no. plays].

In addition to visualization of user interests, integrated presentation of information from Wikipedia, Amazon, YouTube, official sites, and more during conversation will help facilitate meaningful exchange. This system will offer two operation modes, "trace mode" and "timeline mode." Trace mode will allow users to find queries to search for information they feel they needed during conversation with other users, based on the visualized interest information of other users. Timeline mode displays information on the query clicked in chronological order. This function enables users to view videos of interest, encyclopedias, or shopping sites while remaining on the website, and to have other users recommend new content to them. Timeline visualization is proven to be useful for displaying integrated events with chronological information [14], but in this project it will be used to search for information on graphical content while communicating with multiple users.

\section{Structure of Proposed System}

\subsection{System Structure}

The proposed system consists of a data input section, a database section, and a data output section. The data input section will receive user input and RSS information from web services. The database section will structure data and export it in XML format. The data output section will analyze the XML and visualize the information (Fig. 2). The interface consists of: the main panel, which displays interest information(1); the information panel, which displays detailed interest information(2); the picture panel, which displays video sharing website YouTube(3); the icon panel, which displays the pages of encyclopedia website Wikipedia and shopping website Amazon(4); and the communication panel, used to communicate with other users(5) (Fig 3). The main panel will display information in either trace mode or timeline mode (Fig 44). The information panel gives the user detailed information such as top 10 favorite works or chronological tables. The communication panel allows the user to communicate with other users,

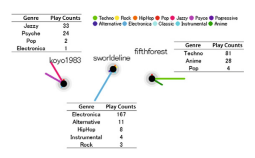

Fig. 1. Design of user node

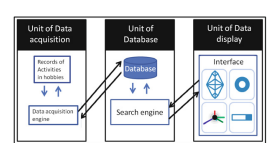

Fig. 2. System overview of SONAR 
based on information found on other panels. Topics are displayed for each query node, making it easy for a user to follow a specific conversation of interest.

\subsection{Trace Mode}

Trace mode allows users to find new queries from a sea of information by referencing the interests of multiple users starting from the user node described in chapter 2.

Structure of Query Nodes. Detailed information users want to know is often spread across various websites such as Wikipedia, YouTube, Amazon, or fan sites, and the necessity to switch pages and search for information obstructs smooth conversation. This system realizes smooth conversation and information acquisition by detecting queries entered during a conversation, making search requests to multiple information websites, and displaying information relevant to the topic integrally on the trace mode interface.

Other than user nodes, this system utilizes query nodes, icon nodes, and timeline nodes (Fig 5). When the user enters a search request into the text box at the top of the screen, a query node is placed in center-screen, with an icon node and timeline node nearby. The letters W, Y, and E on icon nodes represent Wikipedia, YouTube, and E-Commerce website (Amazon, within this article), and clicking on these letters will switch information displayed in the picture panel and icon panel to information related to the keyword. Icon nodes can be set to any website the user likes (e.g. official websites, 2 Channel), and a maximum of 5 can be displayed at any time. Clicking on the timeline node will switch the main panel from trace mode to timeline mode. Node locations are calculated by physical simulation using the spring model, and arranged optimally.

Executing Trace Mode. When the user enters a keyword related to their topic of interest, the database section exports data containing information on query nodes, and the data output section's layout algorithm rearranges these nodes on-screen (Fig 6). For example, if a user takes interest in The Beatles in a conversation and enters the band name into the text box, icon nodes W, Y, E, and timeline node $\mathrm{T}$ will appear around the query node, making reading Wikipedia articles, watching video clips of songs, or checking CD and DVD reviews on Amazon intuitive, meaning the user can easily share this information while continuing their conversation. Clicking on the query node will expand the information panel. If at any time the user feels the screen is cluttered with too many nodes, nodes may be dragged and dropped to other locations.

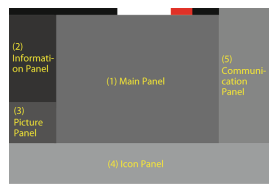

Fig. 3. Overview of user interface

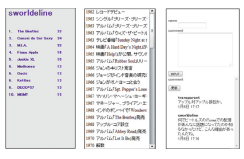

Fig. 4. Left: Information panel that displays the top 10 of taste in music, Center: Information panel that displays the chronology of history, Right: Communication panel 


\subsection{Timeline Mode}

Timeline Objects. Information is often presented in a timeline to make clear their chronological relationship [14]. By placing events in chronological order (Fig 7, right side), the order of these events can be clearly recognized, and by comparing this timeline with other timelines (Fig 7, center), relationships that are hardly noticeable when individually inspected can be discovered and understood. The length and width of timeline objects represent duration and number of events, respectively, with beginning times and dates at the bottom, ends at the top. Timeline mode uses data from Wikipedia.

Executing Timeline Mode. Clicking a query node in trace mode will display multiple events related to that query as a timeline object in chronological order. Clicking on captions in timeline objects will change the chronological table displayed in the information panel. Adding a new query will create a new query node, and an edge will extend to related events within the timeline object. Double-clicking captions in timeline objects will highlight the chronological chart related to the query node (Fig 8 ).

\section{Evaluation Experiment}

In order to investigate whether users with niche interests can gain and exchange more cross-boundary information by using our system, on June 28, 2012, we had 18 subjects - 13 male and 5 female, aged 22 to 26 - who like rock music search for information in any way they please for 30 minutes, then answer the questions on the survey provided on Table 1 from the choices "1: Strongly disagree, 2: Disagree, 3: Neutral, 4: Agree, 5: Strongly Agree." Later, we gave them access to our system for 30 minutes, and had them answer survey questions given on Table 2 to gain their insight. Fig 9 shows the results of the surveys noted in charts 3 and 4, with the green bars representing traditional measures of information acquisition, and the orange bars representing acquisition using the proposed system. The vertical axis shows the average score for each question,

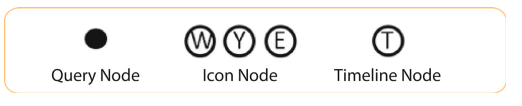

Fig. 5. Definition of each nodes

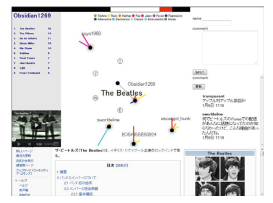

Fig. 6. User interface of trace mode

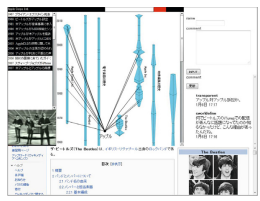

Fig. 7. Timeline objects

Fig. 8. User interface of timeline mode 
and the error bars atop each histogram shows standard deviation. Table 3 shows t-test results. There was a significant difference in the 5\% range for questions 1 and 3, as well as that in the $0.1 \%$ range for question 2 . In other words, the t-test revealed significant difference between information acquisition by traditional methods and our system for all questions. Because our system scores a higher average and lower standard deviation than traditional methods for questions "were you able to find cross-boundary information on your interest?", "did this service help in conversation and exchange of information with other users?", and "would you like to continue to use this service as a method of interest information acquisition?", we conclude that our system is useful in users gaining cross-boundary information on a topic, or exchanging such information.

Furthermore, the $84.2 \%$ rate of subjects choosing answers 4 or 5 for question 4 supports the validity of the method of data presentation by our system. With a spread of 3.78 , answers to question 5 "did user nodes help you find points in common with other

Table 1. Survey questions on information acquisition through previous methods

Q1. Were you able to find cross-boundary information on your interest?

Q2. Did this service help in conversation and exchange of information with other users?

Q3. Would you like to continue to use this service as a method of interest information acquisition?

Table 2. Survey questions on information acquisition through our system (SONAR)

Q1. Were you able to find cross-boundary information on your interest?

Q2. Did this service help in conversation and exchange of information with other users?

Q3. Would you like to continue to use this service as a method of interest information acquisition?

Q4. Did you easy to understand the presentation of data?

Q5. Did user nodes help you find points in common with other users?

Q6. Did query nodes help you understand the relation between objects?

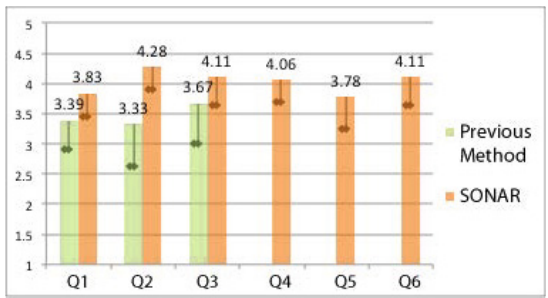

Fig. 9. Mean and standard deviation of the scores in evaluation experiment

Table 3. Result of t-test in evaluation experiment

\begin{tabular}{|c|r|r|r|}
\hline & Q1 & Q2 & Q3 \\
\hline t-values & 2.115 & 4.135 & 1.913 \\
\hline P-values (one-sided test) & 0.0247499 & 0.000346 & 0.036372 \\
\hline
\end{tabular}


users?" show that not every user benefited from the current user node system. As reasons, 2 subjects commented that they "could not find anyone with common interests," and 1 commented that they "used user nodes as a means to distinguish between oneself and others, not as a method to find common interests." However, some users were able to actively utilize user nodes for information acquisition, such as looking back on the music they listened to by viewing the length of colored beam for each genre, or by chatting up users with similar nodes.

The high average score of 4.11 for question 6 "did query nodes help you understand the relation between objects?" suggests the usefulness of nodes when gathering information within niche fields.

In the free comment section, subjects gave feedback such as "Users on the system were all interested in the same topic and thus reacted much more during conversation on the topic, whereas the majority of Twitter users will not," "while existing SNS connect individual users, I felt that the focus on having a common interest facilitated frank communication," "being able to know the interests of other users through user nodes, and learning more about the topic in question was very nice," "being recommended something in a conversation seemed to prompt me to investigate things I previously wouldn't have," and "the strategical connections between objects helped me gain information on cross-boundary connections." These comments are proof that the system achieves its goals of supporting cross-boundary information on interests and facilitation of information exchange between users. The efficacy of the node system, allowing users to discover new queries from a sea of information, is also apparent.

\section{Conclusion}

We have proposed to you a new communication system that helps users gain crossboundary information as well as information between the users themselves. This system allows users to follow nodes to discover potential friends that have similar characteristics of interests, gain information across boundaries, and exchange information with other users. Furthermore, communication with others with similar interests will facilitate social interaction.

we conducted a system evaluation experiment, and the results suggested that our interest-based cross-boundary search and presentation of information is effective for niche fields. Observation of interaction revealed that users were utilizing the icon nodes placed around query nodes to reference YouTube, Wikipedia, Amazon, and other websites in the course of their conversation. Therefore, it can be inferred that our system is effective to a degree when it comes to interest visualization and supporting the acquisition of information based on user interest.

The evaluation experiment revealed that sometimes a user will not be able to find another user with similar interests, especially in cases when the user specifically listened to artists relatively unknown to the extent that nobody else within the subject group knew of them. However, this can be expected from the small size of the subject group, and the likeliness of similar cases will decrease when the system is launched to the public and sees users with a wide range of interests joining, increasing the range of cross-boundary information shared even further. 
In the future, we would like to have many people use our system, so that we can improve the dynamic and specific properties of this service through analysis of compiled usage data.

\section{References}

1. Matsumoto, J.: A Study on Diagnostic Test of the Degree of Health. Journal of Health Science 9, 159-180 (1987) (in Japanese)

2. Tokunaga, M.: Development of Health and Life Habit Inventory (DIHAL. 2). Journal of Health Science 27, 57-70 (2005) (in Japanese)

3. Morimoto, K.: Lifestyle and Health. Journal of the Japan Society of Acupuncture and Moxibustion 53(2), 141-149 (2003) (in Japanese)

4. Suzuki, S., Shigeno, H., Matsumura, K., Kanatsugu, Y.: A Rule-base Contents Conversion Framework for TV Broadcasting Services. IPSJ SIG Technical Report. 2006-ITS-27 120, 185-192 (2006) (in Japanese)

5. Jones, S.: Graphical Query Specification and Dynamic Result Previews for a Digital Library. In: Proceedings of the 11th Annual ACM Symposium on User Interface Software and Technology (UIST 1998), pp. 143-151 (1998)

6. Benford, S., Snowdon, D., Greenhalgh, C., Ingram, R., Knox, I., Brown, C.: VR-VIBE: A Virtual Environment for Co-operative Information Retrieval. Computer Graphics Forum 14(3), 349-360 (1995)

7. Heer, J., Boyd, D.: Vizster: Visualizing Online Social Networks. In: IEEE Symposium on Information Visualization (InfoVis), pp. 32-39 (2005)

8. Nomata, Y., Hoshino, J.: VISTORY: Visualizing Relations of Multi-timelines. The Journal of the Society for Art and Science 7(2), 55-64 (2008) (in Japanese)

9. Robertson, G.G., Mackinlay, J.D.: The Document Lens. In: Proceedings of the 6th Annual ACM Symposium on User Interface Software and Technology (UIST 1993), pp. 101-108 (1993)

10. Mackinlay, J.D., Robertson, G.G., Card, S.K.: The Perspective Wall: Detail and Context Smoothly Integrated. In: Proceedings of the SIGCHI Conference on Human Factors in Computing Systems (CHI 1991), pp. 173-176 (1991)

11. Sarkar, M., Brown, M.H.: Graphical Fisheye Views. Communications of the ACM 37(12), 73-83 (1994)

12. Johnson, B., Shneiderman, B.: Tree-maps: A Space-filling Approach to the Visualization of Hierarchical Information Structures. In: Proceedings of IEEE Conference on Visualization, pp. 284-291 (1991)

13. newsmap, http://newsmap.jp/

14. Tufte, E.R.: The Visual Display of Quantitative Information, 2nd edn. Graphic Press (2001) 